Risto Paju

\title{
EIN FUND IN DER MUSEUMSSAMMLUNG. EIN HEILIGER JAKOBUS AUS KALKSTEIN IN DER SAMMLUNG BEHAUENER STEINE DES TALLINNER (REVALER) STADTMUSEUMS
}

Iacobe servorum spes medicina tuorum, alleluia, alleluia. ${ }^{1}$

In der Sammlung behauener Steine des Tallinner Stadtmuseums ${ }^{2}$ befindet sich eine aus Kalkstein vom Lasnamägi (Laaksberg) ${ }^{3}$ gehauene, 52,5 $\mathrm{cm}$ hohe Skulptur (Abb. 1). Diese stellt einen schmalen Mann dar in langer Robe mit Stab und mit einer am Hals hängenden Reisetasche. Die Figur hat bedauerlicherweise sehr gelitten. Zusätzlich zur demolierten Vorderseite fehlt ihr der Kopf - erhalten ist einzig ein Teil des Barts.

Ziel des vorliegenden Artikels ist es, diese bisher nicht behandelte Skulptur näher zu betrachten, um wen handelte es sich, wo könnte sie einst gestanden haben, und die auf ihr befindlichen Farbreste zu untersuchen. Der Anreiz zum Verfassen dieses Beitrags bestand vor allem darin, dass mittelalterliche, rundplastische Steinskulpturen in Estland

DOI: http://dx.doi.org/10.12697/BJAH.2016.12.07

Übersetzung aus dem Estnischen von Marju und Olaf Mertelsmann.

Anm. d. Übers.: Historische Ortsnamen werden vor 1918 in ihrer deutschen Form wiedergegeben, soweit möglich, danach in ihrer estnischen Fassung.

1 Liber Sancti Jacobi oder Codex Calixtinus (12. Jahrhundert): Hoffnung und Besserung deinen Dienern Jakobus! Halleluja, halleluja.

2 Ausführlicher über die Sammlung siehe: Risto Paju, „Otsides kivist jumalat. Ühest figuraalse kujutisega raidkivifragmendist linnamuuseumi kogus”, Vana Tallinn, 26 (30) (Tallinn: Tallinna Linnaarhiiv, 2015), 173-174.

3 Der Autor bedankt sich bei Helle Perens für die Hilfe beim Bestimmen des Gesteins. 


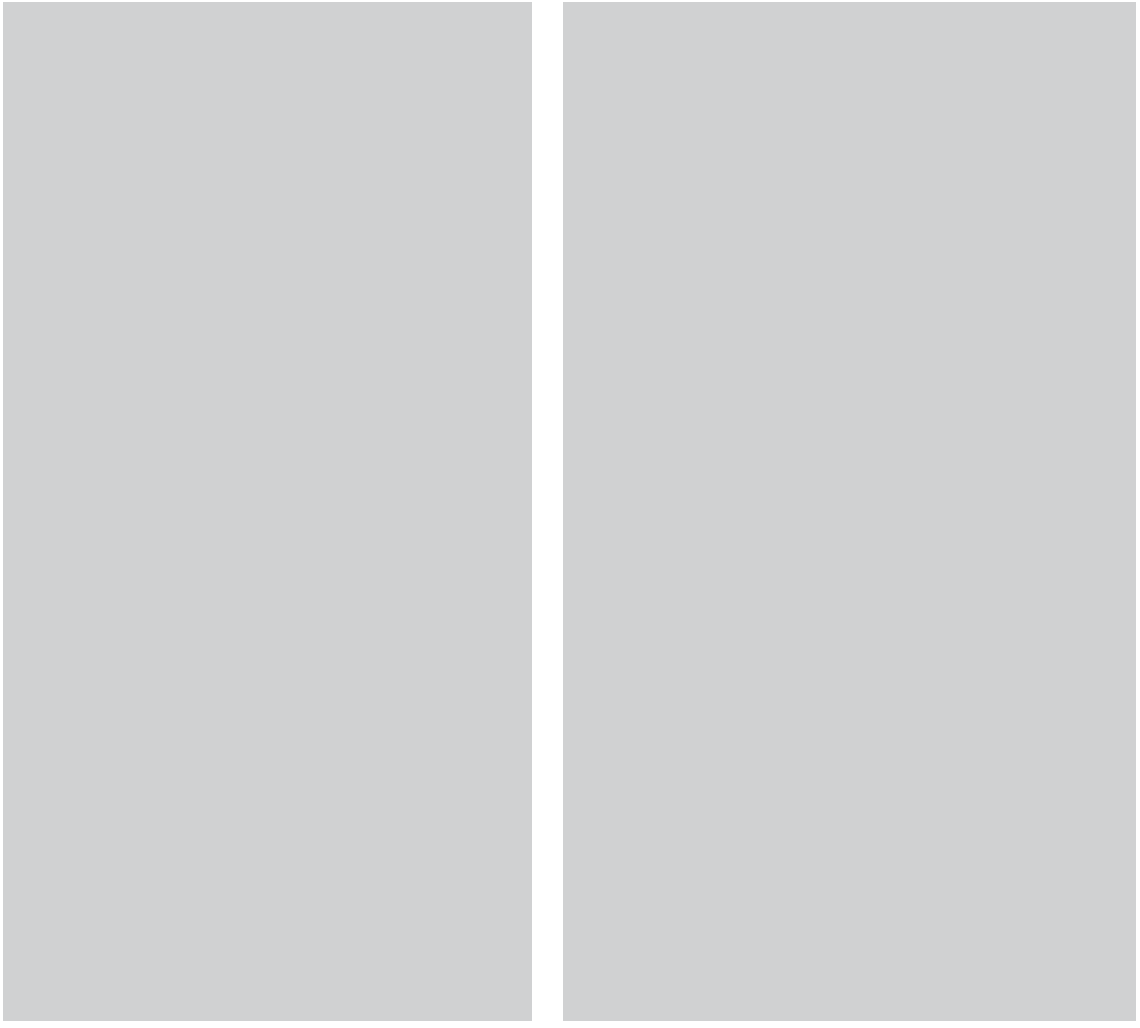

Abb. 1. Ein Heiliger Jakobus aus Kalkstein in der Sammlung behauener Steine des Tallinne (Revaler) Stadtmuseums. Fotograf: Risto Paju.

Abb. 2. Heiliger Jakobus. Fragment. Fotograf: Risto Paju.

nur sehr wenig erhalten sind. Ebenso wurde diese Figur in früheren Publikationen nicht behandelt oder auch nur erwähnt.

Zuerst kann bestätigt werden, dass die Skulptur ungeachtet des Verlorengegangenen derart ganzheitlich ist, dass es möglich ist zu verstehen, wer dargestellt wird. Wie erwähnt befindet sich in der rechten Hand des Mannes ein Spazierstock oder ein Stab und in der linken, wie nach dem erhaltenen Fragment vermutet werden kann, ist ein Buch gewesen. An der teilweise erhaltenen Schultertasche aber sehen wir die Figur einer Kammmuschel (Abb. 2). Stab und Kammmuschel sagen uns, dass es sich um eine Figur des Heiligen Jakobus handelt.

Der Kult des Heiligen Jakobus in Reval verdient eine eigene Untersuchung, in diesem, eine konkrete Musealie betrachtenden Aufsatz berühre ich dieses Thema einzig im Vorübergehen. Über seine Verehrung im Raum der Hanse wurde bereits geschrieben: als Patron der Kaufleute und besonderer Patron der Hanse wird der Heilige Jakobus der Ältere in schriftlichen Quellen häufig erwähnt, der im Hanseraum besonders verehrt wurde. ${ }^{4}$ Ebenso ist an anderer Stelle auch gesagt worden, dass früher zwischendurch behauptet wurde, dass die Hanse unter der Patronage des Apostels Jakobus des Älteren gestanden hätte. Dies entsprich sicherlich nicht der Realität, denn die Hanse verfügte über keinen gemeinsamen Schutzheiligen. ${ }^{5}$

Es handelte sich zweifelsohne um einen wichtigen katholischen Heiligen und Wallfahrten nach Santiago de Compostela unternahmen auch Revaler. Beispielsweise ist bekannt, dass 1486-1487 der Revaler Kaufmann Bernd Pal eine Wallfahrt dorthin unternahm. ${ }^{6}$

Heutzutage finden sich in Tallinn mittelalterliche skulpturale Figuren des Heiligen Jakobus noch beispielsweise auf dem Flügelaltar der Niguliste kirik (Nikolaikirche) und auf dem Altar der Püha Vaimu kirik (Heiligengeist-Kirche), wo diese aus Holz gefertigt sind. ${ }^{7}$ Die Tatsache, dass in Tallinn auch ein in Stein gehauener Jakobus erhalten ist, erweitert den Kreis der mittelalterlichen Figuralskulpturen.

Vor einer näheren Betrachtung der Skulptur muss noch auf die Herkunft und den Fundort eingegangen werden. Irgendwann in der zweiten Hälfte der siebziger Jahre des 20. Jahrhunderts wurde diese in der Niguliste tänav (Nikolai-Str.) $22^{8}$ (Abb. 3) im Keller inmitten von Müll ${ }^{9}$ von der Kunsthistorikerin Silvi Lindmaa-Pihlak gefunden, die damals architekturhistorische Untersuchungen im Haus ausführte. Für viele Jahre befand sie sich in der Restaurierungsverwaltung der Republik. Ins Museum gelangte die Skulptur durch die Vermittlung des Architekturhistorikers Sulev Mäeväli im Jahr 1995. ${ }^{10}$

\footnotetext{
4 Matthias Zender, „Heiligenverehrung im Hanseraum”, Hansische Geschichtsblätter, Bd. 92 (Köln, Wien: Böhlau Verlag, 1974), 11.

Hedwig Röckelein, „Die Verehrung des Apostels Jakobus d. Ä. in den norddeutschen Hansestädten. Eine Einführung”, Jakobus-Studien, Der Kult des Apostels Jakobus d. Ä. in norddeutschen Hansestädten hrsg. von Hedwig Röckelein (Tübingen: Gunter Narr Verlag, 2005), 18.

6 Carsten Jahnke, „Bernd Pal, ein Kaufmann des 15. Jahrhunderts. Eine biographische Skizze”, Vana Tallinn, 15 (19) (Tallinn: Estopol, 2004), 162.

7 Villem Raam, Gooti puuskulptuur Eestis (Tallinn: Kunst, 1976), 42 und 48-53.

8 Grundstück Nr. 571 .

9 Tallinna Linnamuuseum / Tallinner Stadtmuseum [TLM] 24498 RMT wissenschaftliche
}

TLM Annahme-Akt Nr. 26. 23.10.1995. 


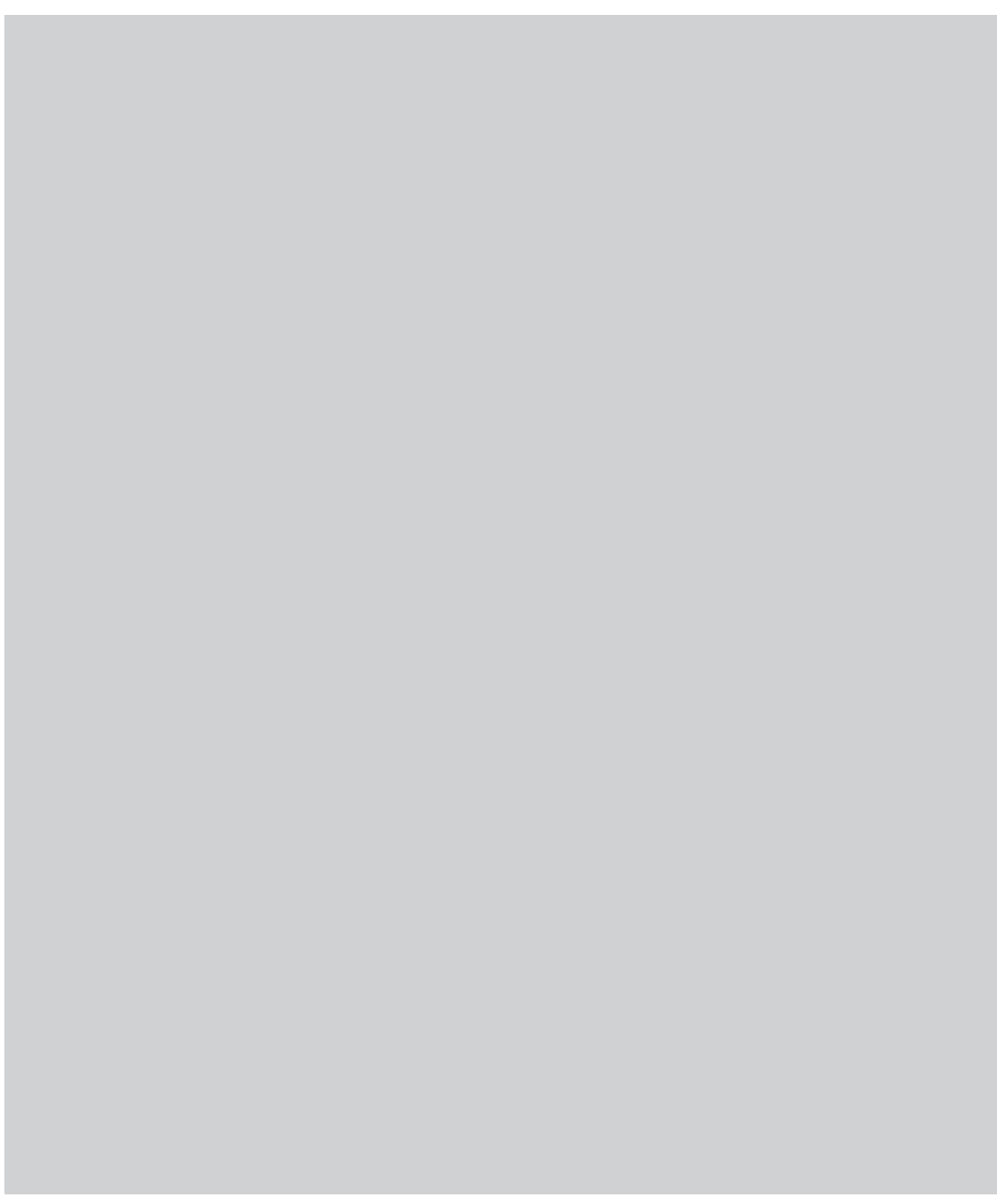

Abb. 3. Niguliste tänav (Nikolai-Str.) 22. Das Gebäude, aus dessen Keller die Statue gefunden wurde. Fotograf: Risto Paju.

Über das frühere Schicksal der Jakobus-Figur ist aber nichts bekannt. Es erscheint als möglich, dass diese von Anfang an aus demselben Haus stammt, doch ist es auch möglich, dass die Figur von anderswo in den Keller geraten war. Die einfachste und wahrscheinlichste Variante besteht darin, dass sie aus demselben Gebäude stammt, doch auch die zweite Möglichkeit ist dennoch nicht ausgeschlossen.

\section{DIE SKULPTUR IN DER NÄHEREN BETRACHTUNG}

Stilistisch gehört die Skulptur zur Gotik und die Figur kann stilkritisch auf das Ende des 15. oder den Beginn des 16. Jahrhunderts datiert werden. Für eine der ältesten erhaltenen rundplastischen Steinfiguren in Estland wird die im westlichen Portal im Wimperg der Kirche in Ridala (Röthel) befindliche Heilige Maria Magdalena aus dem 13. Jahrhundert gehalten. ${ }^{11}$ Einen zusätzlichen Wert verleiht die Tatsache, dass sie sich in situ befindet. Auch der Heilige Jakobus könnte anfangs in einer Nische gestanden haben. Darauf mag schon das oben erwähnte Detail hinweisen, dass der Rückenteil der Figur unbearbeitet ist und dass am Rücken ein Metallteil befestigt ist, mit dessen Hilfe die Skulptur beispielsweise an der Wand eines Gebäudes fixiert werden könnte.

Aufmerksamkeit erregt das Material der Sklulptur - Kalkstein vom Lasnamägi. Feinere Arbeiten wurden in Reval mehrheitlich aus Dolomit aus Orgita angefertigt. Kalkstein vom Lasnamägi ist in Tallinn vor allem bekannt als Material von Mauersteinen und behauenen Steinen einfacherer Form (Gewölberippen, Konsolen u. a. m.). Aus diesem Stein sind wohl auch ornamental anspruchsvollere Arbeiten angefertigt worden, doch durch seine Eigenschaften ist der Stein aus Orgita für diese Arbeit besser geeignet. Mit dieser hier vorgestellten Arbeit der Steinbildhauerei ist der Kalkstein vom Lasnamägi aber in den Status von Skulpturenmaterial erhoben worden. Die älteste bekannte Nutzung des Dolomitgesteins aus Orgita erfolgte in Reval im 14. Jahrhundert, als daraus der Schlussstein des Chorraums der Domkirche (Toomkirik) gefertigt wurde. ${ }^{12}$ Der genaue Zeitpunkt, wann Dolomit aus Orgita in der Unterstadt im Baubild erschien, ist bisher unmöglich gewesen festzustellen. Eine exakte Datierung von Steinmetzarbeiten aus Dolomit aus Orgita in der Unterstadt stammt vom Beginn des zweiten Jahrzehnts des 16. Jahrhunderts. ${ }^{13}$ Möglich, dass zum Zeitpunkt der Erzeugung von Jakobus die Nutzung dieses Dolomitgesteins noch nicht weit verbreitet war.

11 Eesti kunsti ajalugu 1. Eesti kunst kõige varasemast ajast kuni 19. saj. keskpaigani (Tallinn: Kunst

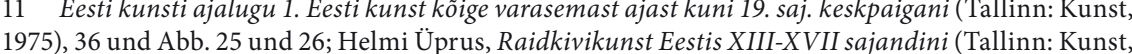
1975), 36 und Abb. 25 und 26; Helmi Uprus, Raidkivikunst Eestis XIII-XVII sajandini (Tallinn: Kuns,
1987 und Abb. 9 und 10.

12 Helle Perens, Paekivi Eesti ehitistes IV. Tallinn (Tallinn: Eesti Geoloogiakeskus, 2010), 22 und

3 Perens, Paekivi Eesti ehitistes IV. Tallinn, 66 


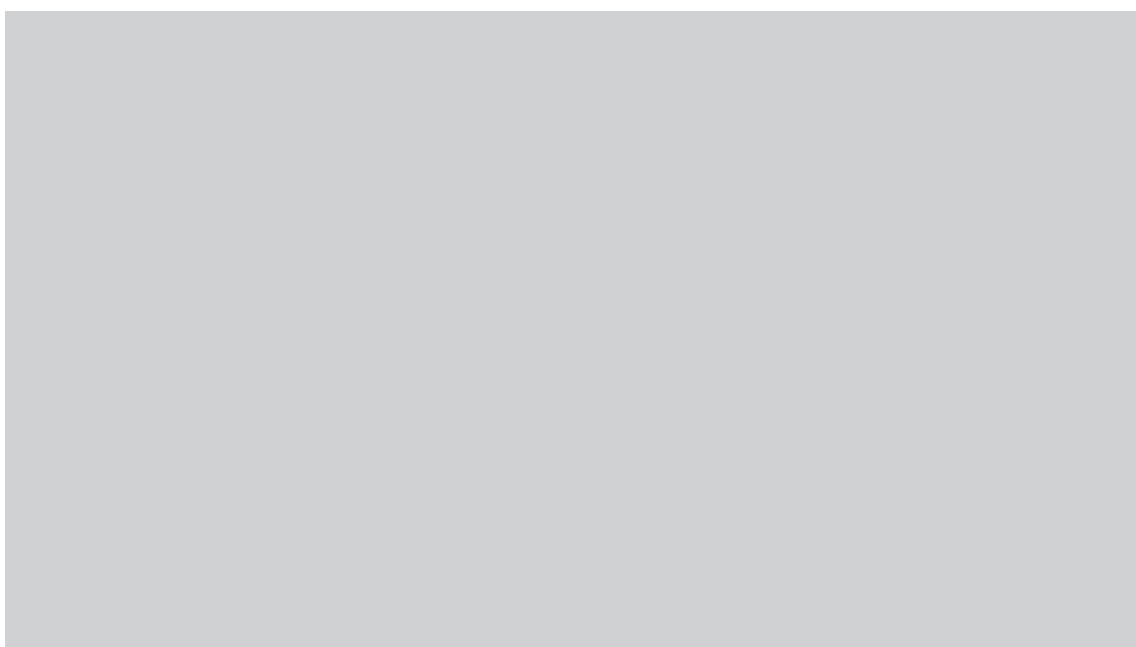

Abb. 4. Heiliger Jakobus. Fragment. Fotograf: Risto Paju.

Mit dem Gestein vom Lasnamägi ist ein gutes Ergebnis erzielt worden, die Faltenstruktur der Robe des Heiligen Jakobus und die erhalten gebliebenen Bartfragmente sind ausgesprochen fein ausgehauen. Besonders sticht bei der rechten, den Stab haltenden Hand der drapierte, wehende Mantel ins Auge (Abb. 4). Leider ist das Gesicht der Skulptur nicht erhalten.

Die Figur ist polychrom gewesen. Offensichtlich ist diese auch nach der Entfernung von ihrem Standort unter verhältnismäßig günstigen Bedingungen aufbewahrt worden und die gesamte Farbschicht ist nicht komplett zerstört, dennoch ist davon nur noch wenig vorhanden. Mit bloßem Auge lassen sich Fragmente rötlicher Farbe erkennen. Für diesen Aufsatz entnahm die Konservatorin des Tallinner Stadtmuseums, Annely Miil, der Skulptur zwei Farbproben. Aus ihnen wurden in der Restaurierungswerkstatt Kanut zwei Mikroschnitte angefertigt. ${ }^{14}$ Die erste Probe wurde vom unteren Teil der Figur neben dem Bein entnommen und die zweite vom Riemen der Schultertasche (Abb. 5 und 6). In der ersten Probe wurden Reste roter Farbe, in der zweiten Überbleibsel von dunkelblauer (?), roter und hellblauer Farbe gefunden. ${ }^{15}$

14 Die Mikroschnitte fertigte Kristina Aas an, den Bericht stellte Heige Peets zusammen.

15 Bericht über die Anfertigung von Mikroschnitten. Objekt: Skulptur Jakobus TLM 24498 . Erstell von Heige Peets, SA EVM Konserveerimis- ja digiteerimiskeskus KANUT. Tallinn 2015.
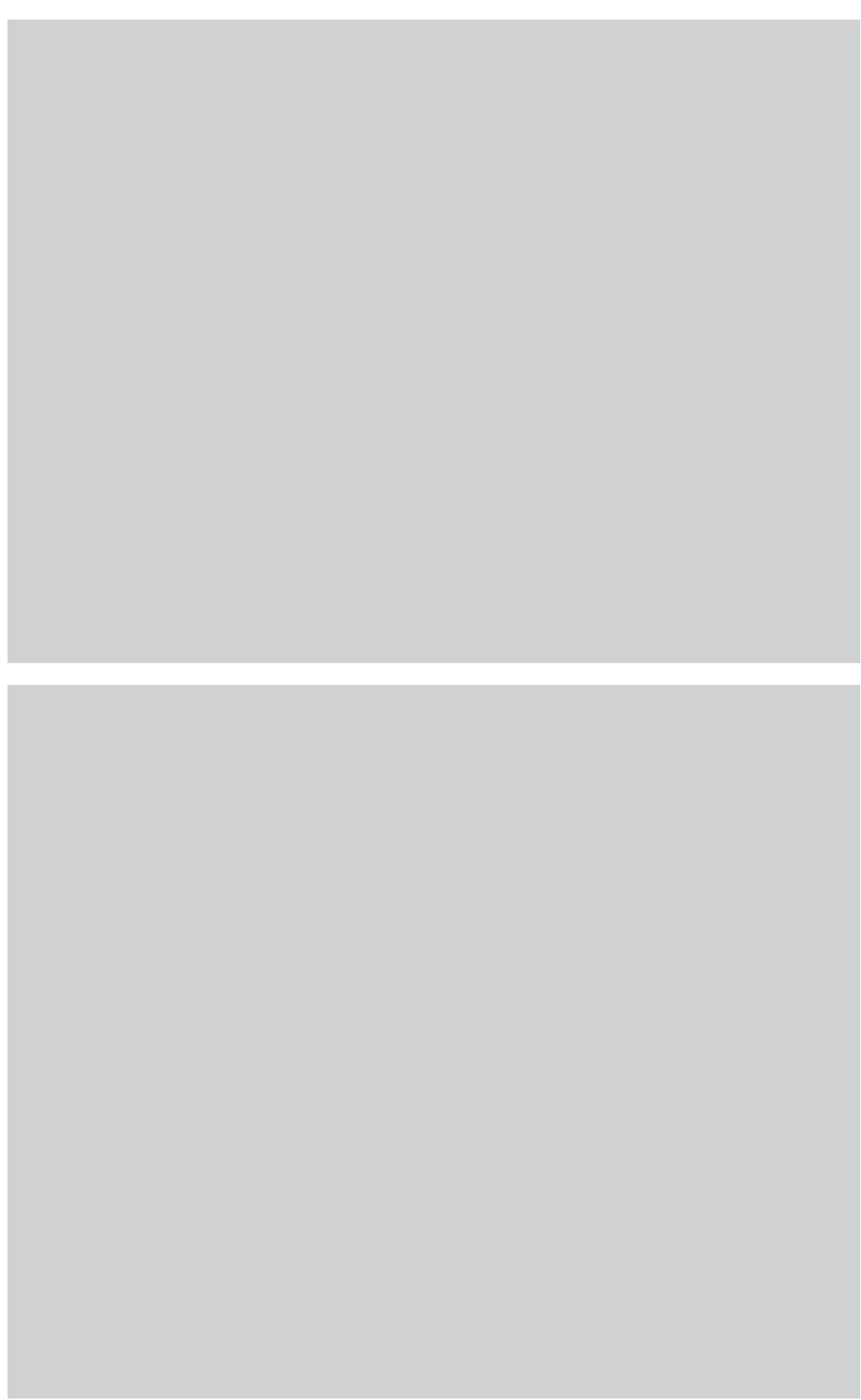

Abb. 5. Heiliger Jakobus. Fragment. Fotograf: Annely Miil. Abb. 6. Heiliger Jakobus. Fragment. Fotograf: Annely Miil. 


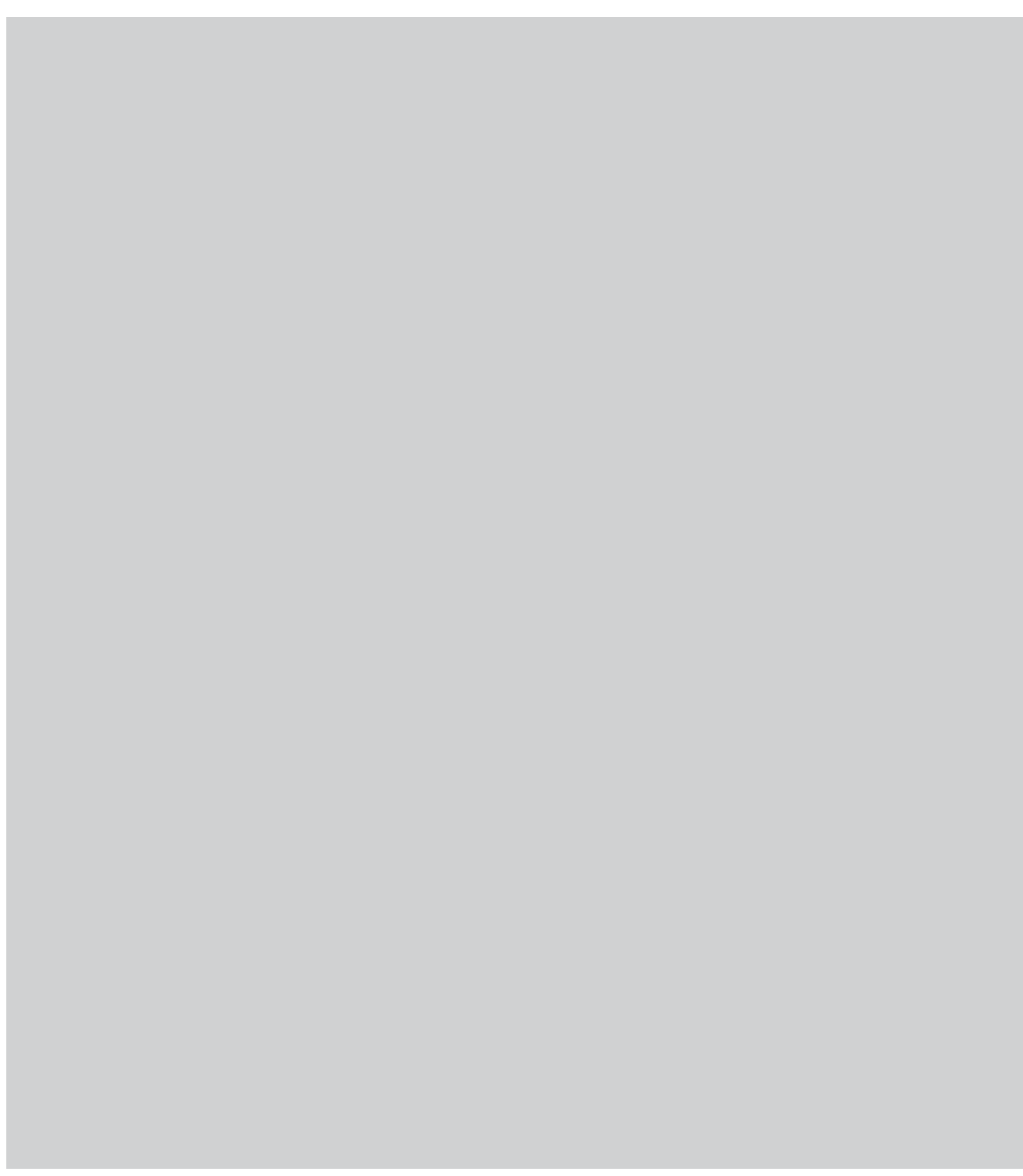

Abb. 7. Lühike jalg (Kurzer Domberg) 1 / Rüütli (Ritterstr.) 2 vor dem Umbau. Estnisches Geschichtsmuseum, Tallinn.

Nach den Worten der Konservatorin Annely Miil kann die laut des Berichts der Mikroschnitte sichtbare Abwechslung von Leim-, Grundierungs- und Farbschichten darauf hinweisen, dass die Skulptur nicht dafür gedacht war, sich unter offenem Himmel zu befinden, sondern in Innenräumen, ${ }^{16}$ doch es kann vermutet werden, dass sich eine Nische, die gegen das Wetter verteidigt, nicht ausschließen lässt.

16 Schreiben Annely Miils an den Verfasser vom 8. März 2016.
Als Zusammenfassung der Beschreibung kann gesagt werden, dass der zu betrachtende Jakobus in dem ihn umgebenden Kontext ein ziemlich ins Auge stechendes skulpturelles Werk darstellt und von einem ausgesprochen kunstfertigen Meister hergestellt worden sein muss.

\section{DER URSPRÜNGLICHE STANDORT?}

Für welchen Ort könnte eine solche Figur gefertigt worden sein? Es lässt sich nicht ausschließen, dass die Heiligenfigur aus einem Sakralbau stammt ${ }^{17}$ doch auch Wohnhäuser konnten Heilige enthalten. Beispielsweise an der Außenfassade oder auch irgendwo in den Innenräumen.

Eine Fassadennische, über die vermutet werden kann, dass sie angefertigt wurde für die Skulptur eines Heiligen und wo sich heute tatsächlich als Schöpfung eines zeitgenössischen Bildhauers eine Figur, welche die Heilige Maria mit Kind darstellt, befindet sich schräg gegenüber von jenem Haus in der Niguliste tänav 22 an der Ecke Rüütli tänav (Ritterstr.) 2 / Lühike jalg (Kurzer Domberg) 1 (Abb. 7 und 8). Dies führt zur Frage, ist es möglich, dass der Jakobus aus dieser Nische stammt? Dies kann nicht ausgeschlossen werden. Auf die Nähe des Fundorts weist direkt wohl nichts hin.

Um festzustellen, ob die zu betrachtende Jakobus-Skulptur in diese Kalksteinnische passt, führten der Verfasser und der Architekt Indrek Laos im Winter 2016 in der Nische, die sich im ersten Stockwerk befindet, Vermessungsarbeiten durch. Nach den Angaben der Messung fertigte der Architekt eine digitale Rekonstruktion an, die zeigt, dass dies grundsätzlich möglich wäre. ${ }^{18}$ (Rekonstruktionsversuch Abb. 9)

Für den Fundort, das Haus in der Niguliste tänav 22, ist es bis jetzt noch nicht geglückt festzustellen, wie dessen mittelalterliches Erscheinungsbild war. Auf einer Fassadenzeichnung aus dem Jahr 1825 ist das Gebäude schon in umgebauter Form zu sehen. ${ }^{19}$ Auch in Archiven ist es nicht geglückt, entsprechende Materialien zu finden. ${ }^{20}$ Deshalb fehlen momen$\tan$ Angaben darüber, ob dieses Haus in früherer Zeit an der Fassade

17 In der Nähe befindet sich auch die Nikolaikirche.

18 Zwischen der Figur und dem Gesims verbleiben für den Kopf $12,5 \mathrm{~cm}$

9 Helmi Üprus, Tallinn aastal 1825 (Tallinn: Kunst 1965), Tafel VII.

Nachfragen im Archiv: Schreiben Kalmer Mäeorgs (Tallinner Stadtarchiv, Tallinna Linnaarhiiv) J Jagos (Estnisches Historisches Archiv, Eest Ajalooarhive) an den Verfasser vom 23. November 2015. 


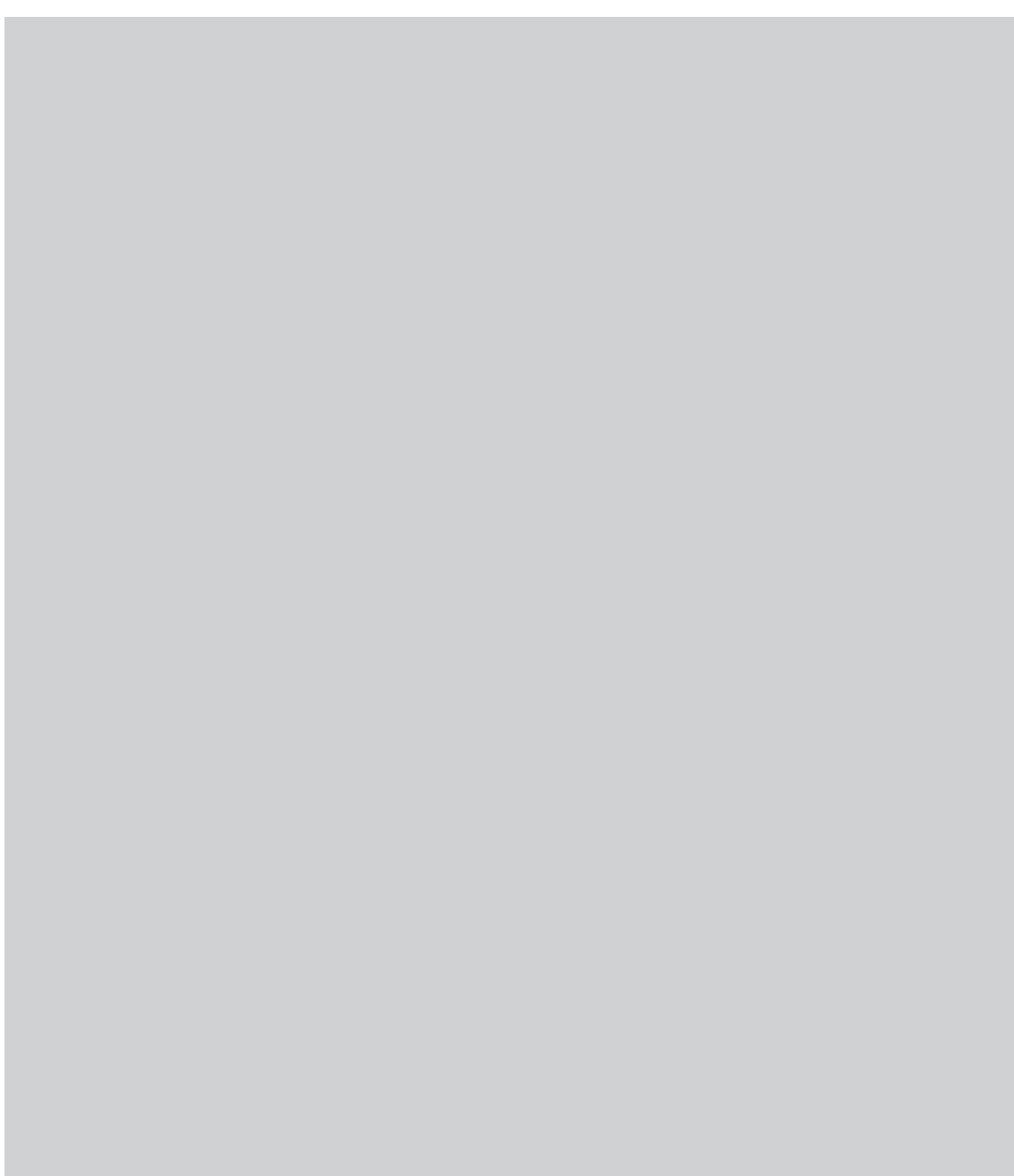

Abb. 8. Heilige Maria mit dem Kind zu finden unter der Lühike jalg (Kurzer Domberg) 1 / Rüütli (Ritterstr.) 2. Fotograf: Risto Paju.

Heiligenbilder oder in ihr eine für eine Heiligenfigur gedachte Nische aufwies.

In dem Fall, dass es sich beim Fundort der Skulptur um ihren ursprünglichen Standort handelt, lautet dann die Frage, zu welcher Zeit wurde die Skulptur in Auftrag gegeben? Unter den Besitzern des 15. Jahrhunderts oder des frühen 16. Jahrhunderts findet sich kein einziger

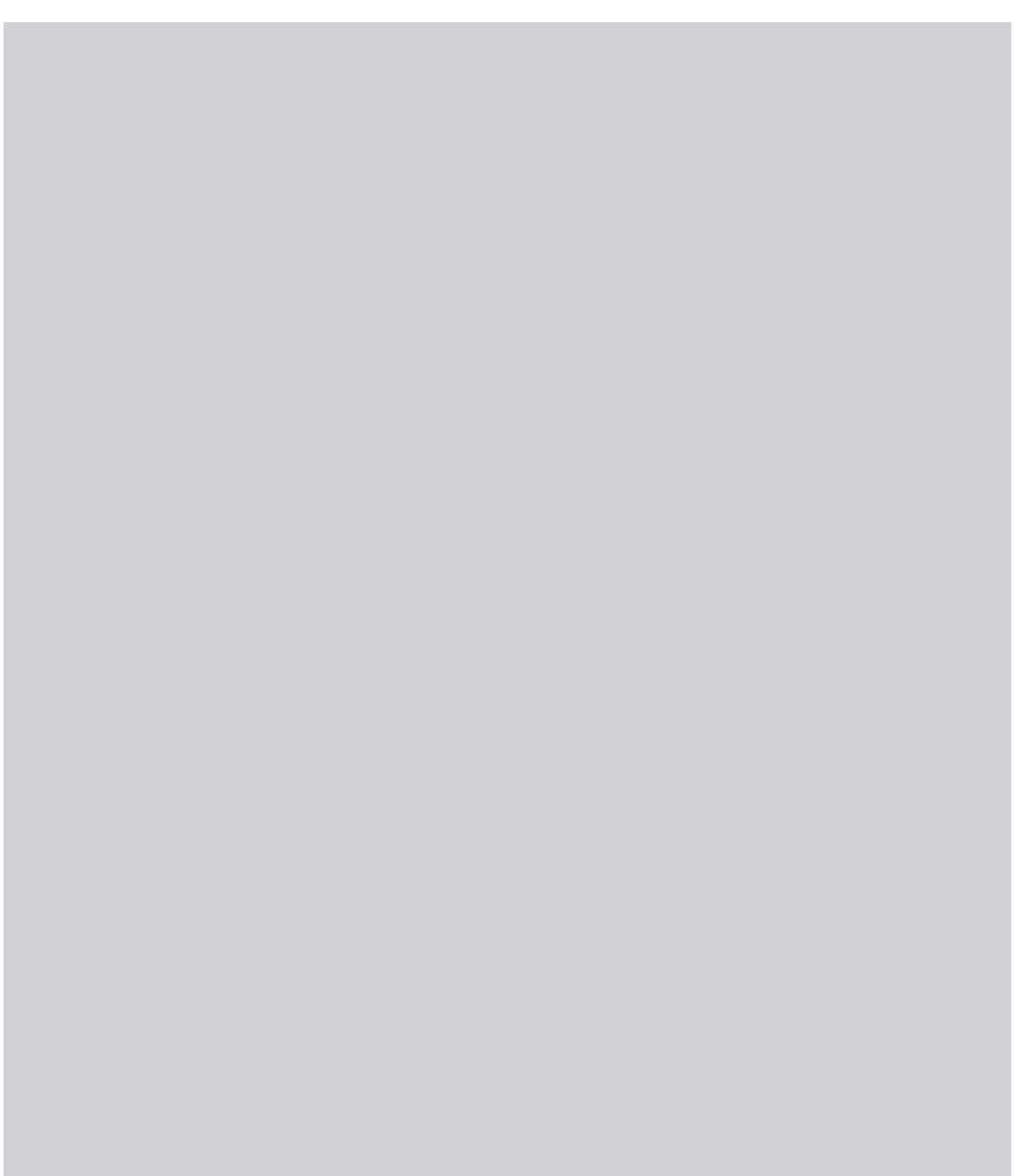

Abb. 9. Rekonstruktion durch Indrek Laos. Flux Projekt.

Jakob. ${ }^{21} 1473$ gehörte das Haus schon dem Maler Clawes Sittow, doch es lebte ein Hans Wagehals drinnen. ${ }^{22}$ Es gibt aber keine Grundlage, die Skulptur mit dem Namen Clawes Sittows zu verbinden.

21 Leo Tiik, Väljavõtted Tallinna kinnisturaamatutest (Handschriftliches Exemplar in der Bibliothek des Tallinner Stadtmuseums), 331-332.

22 Ibidem, 331 
Dies, dass es sich beim Jakobus um eine in der Fassade eines Gebäudes befindliche Heiligenfigur handelte, bleibt verständlicherweise eine Vermutung oder eine Möglichkeit und die Skulptur kann möglicherweise auch Bestandteil einer Innenausstattung gewesen sein. Auf die eine oder andere Weise ist dies eine zusätzliche Information über die Kunst des mittelalterlichen Revals, denn durch die Zeiten hindurch sind alle derartigen gemalten, geformten oder gehauenen Heiligen oder andere Szenen aus den Fassadennischen oder andernorts überwiegend verschwunden. Erhalten geblieben ist sehr wenig und dies mehrheitlich erst aus dem Spätmittelalter oder bereits aus der frühen Neuzeit.

Von den Heiligen ist beispielsweise das aus der Fassade des Hauses der Kanutigilde stammende und das Datum 1516 tragende Relief mit der Figur des Heiligen Kanuti erhalten geblieben, gefertigt aus Dolomit aus Orgita. ${ }^{23}$ In der Fassade des benachbarten Hauses der Olafsgilde ist ein Relief des Heiligen Olaf erkennbar.

Als Beispiel können noch Tafelmalereien von einem Haus in der Kuninga tänav (Königsstr.) 1 angeführt werden (1530-155024). Die Fassade verzieren heutzutage Kopien, die Originale befinden sich in der Kunstsammlung des Tallinner Stadtmuseums. ${ }^{25}$ An Tafelmalereien ist auch ein Gemälde mit der Szene des Jüngsten Gerichts erhalten, das sich an der Fassade des Rathauses befindet. ${ }^{26}$ Datiert wird dies auf die Jahre 1530-1540.27 Das Original findet sich im Tallinner Stadtmuseum und an der Fassade des Rathauses ist eine Kopie. Nischen oder Nischenfragmente, in denen ein Gemälde oder eine Skulptur gewesen sein könnte, finden sich in Tallinn an mehreren Orten. Beispielsweise ist in der Pikk tänav (Langstr.) 29 teilweise eine Nische mit dreieckigem Oberteil erhalten, die sich einst direkt am Ort des Außentürportals im gotischen Stil befand und offensichtlich mit diesem eine ganzheitliche Komposition bildete. An der Ecke von Tolli tänav (Zollstr.) und der Pikk tänav mit der Anschrift Tolli tänav 2 liegt ein als die „drei Schwestern” bekannter

23 Heute im Niguliste-Museum aufbewahrt; Merike Kurisoo, Isabel Aaso-Zahradnikova, „Kivist kuningas Kanut. Tallinna Kanuti gildi maja hiliskeskaegsest pühakureljeefist ja selle tehnilistest uuringutest", Eesti Kunstimuuseumi Toimetised, 2 [7] (2012), 168.

24 Krista Kodres, Esitledes iseend. Tallinlane ja tema elamu varauusajal (Tallinn: Tallinna Ülikooli kirjastus, 2014), 68.

25 TLM 19857 G, 19858 G, 19859 G, 19860 G, 19861 G, 19862 G.

26 TLM $9322 \mathrm{G}$.

Krista Kodres, „Avalikud hooned - omavalitsuste arhitektuurne ja kunstiline manifest. Tallinna raekoda", Eesti kunsti ajalugu II köide 1520-1770 (Tallinn: Eest Kunstiakadeemia, 2005), 72.
Gebäudekomplex. Am gotischen Portal des der Tolli tänav am stärksten zugewandten Hauses lassen sich ebenfalls die Überbleibsel eine ehemaligen Nische erkennen. Die Nische in ihrer Ganzheit ist aber auf einer Zeichnung von Ch. A. Gabler aus dem Jahr 1856 gut zu sehen, auf der das äußere Erscheinungsbild des Gebäudes vor dem Umbau dargestellt ist. ${ }^{28}$ Ebenso bildet dies auch E. H. Schlichtung auf seinem Gemälde der Ecke der Langstr. und der Zollstr. ab, welches aus der Mitte des 19. Jahrhunderts stammt. ${ }^{29}$ Im Endgiebel der Vene tänav (Russische Str.) 12 können wir zwei runde, jetzt leere Nischen sehen usw.

Zusammenfassend kann gesagt werden, dass über den ursprünglichen Standort der Skulptur nur Vermutungen angestellt werden können, was ein Ziel dieses Beitrags ist, doch ausgesprochen sichere Antworten können nicht gegeben werden.

\section{ZUSAMMENFASSUNG}

Die stilistisch zur Gotik zählende Skulptur stammt wahrscheinlich aus dem 15. oder dem frühen 16. Jahrhundert. Mit keinem der Besitzer des Hauses in der Niguliste tänav 22 lässt sie sich momentan verbinden beispielsweise lässt sich unter ihnen, soweit überliefert, keiner finden, der den Vornamen Jakob getragen hätte und als dessen Namenspatron der Heilige geeignet wäre. ${ }^{30}$

Über die Figur kann gesagt werden, dass es sich um die kunstvolle Arbeit eines qualifizierten Meisters handelt, die ungeachtet dessen, dass es sich bei Kalkstein vom Lasnamägi nicht um das beste Material für Skulpturen handelt, fein gehauen und gut proportioniert ist. Teilweise ist auf der Oberfläche der Skulptur auch Polychromie erhalten.

Alle Interessierten können die Skulptur in der Ausstellung im neuen Hauptgebäudes des Estnischen Nationalmuseums (Eesti Rahva Muuseum) betrachten

aastal 1825,17

Tiik, Väljavõtted Tallinna kinnisturaamatutest, Grundstück Nr. 571 
Risto Paju: A New Find From A Museum Collection: The Limestone St James From The Collection Of Ashlars At The Tallinn City Museum Keywords: Medieval Tallinn; St James; Sculpture; Gothic; Collection OF Ashlars At The Tallinna City Museum

\section{SUMMARY:}

The Collection of Ashlars at the Tallinn City Museum includes a sculpture that stands $52.5 \mathrm{~cm}$ tall and is hewn from Lasnamägi limestone (Abb. 1). It depicts a slender man in a long robe, with a staff and travel bag around his neck. The statue has been seriously damaged. In addition to the broken front, its head is also missing - all that has survived is part of its beard. The goal of the article is to more closely examine the sculpture, which has not received any attention to date, in order to determine who it depicts, where it could be been located, and examine the surviving traces of paint. The primary motivation for writing the article is the fact that very few medieval three-dimensional stone sculptures have survived in Estonia. Also, this figure has not been dealt with or even mentioned in earlier writings.

It can be said that, regardless of the damage it has suffered, the sculpture is complete enough to determine who is depicted. As stated above the man has a walking stick or staff in his right hand and, based on the surviving fragments, a book in his right hand. On the partially surviving bag, we see the image of a scallop (Abb. 2). The staff and scallop tell us that this is a figure of St James. The sculpture is gothic in style, and based thereon, it can be dated back to the $15^{\text {th }}$ or early $16^{\text {th }}$ century. The sculptural material - Lasnamägi limestone - arouses attention.

Where was this sculpture to be placed? We cannot dismiss the fact that the figure of the saint comes from a sacral building, but there could also have been saints in residential buildings, for example, on exterior facades or somewhere indoors. In summary, one must admit that the original location of the sculpture can only be speculated upon and definite answers are not possible. However, what is certain is that this is the work of a skilled master. Regardless of the fact that Lasnamägi limestone is not the best sculptural material, the work is finely hewn and well-proportioned. The sculpture is now on display in the new Estonian National Museum building.
CV:

Risto Paju has been a curator and keeper of the Collection of Ashlars at the Tallinn City Museum since 2005. His research is focused on the exhibits in the Collection of Ashlars at the City Museum, based on which he has published articles and organised exhibitions. Of the latter, the most important the permanent exposition in the Carved Stone Museum, which he curated in 2016. Paju has also dealt with researching the inventory lists of Tallinn's $18^{\text {th }}$ century citizenry. 
